\title{
Global Well-Posedness of the Inviscid Heat-Conductive Resistive Compressible MHD in a Strip Domain
}

\author{
Yanjin Wang ${ }^{1}$ and Zhouping Xin ${ }^{2, *}$ \\ ${ }^{1}$ School of Mathematical Sciences, Xiamen University, Xiamen, Fujian \\ 361005, China. \\ 2 The Institute of Mathematical Sciences, The Chinese University of \\ Hong Kong, Shatin, NT, Hong Kong, SAR, China.
}

Received 10 June 2021; Accepted 12 August 2021

\begin{abstract}
This paper concerns the inviscid, heat-conductive and resistive compressible MHD system in a horizontally periodic flat strip domain. The global well-posedness of the problem around an equilibrium with the positive constant density and temperature and a uniform non-horizontal magnetic field is established, and the solution decays to the equilibrium almost exponentially. Our result reveals the strong stabilizing effect of the transversal magnetic field and resistivity as the global well-posedness of compressible inviscid heat-conductive flows in multi-D is unknown.
\end{abstract}

AMS subject classifications: 35A01, 35Q35, 76N10, 76W05

Key words: Compressible MHD, inviscid heat-conducting flow, magnetic diffusion, global well-posedness, strip domain.

\section{Introduction}

When the viscosity is neglected whereas the heat conduction and magnetic diffusion are taken into account, the dynamics of compressible electrically conducting

${ }^{*}$ Corresponding author. Email addresses: yanjin_wang@xmu.edu.cn (Y. J. Wang), zpxin@ims. cuhk.edu.hk (Z. P. Xin) 
fluids interacting with magnetic fields can be described by the following magnetohydrodynamic system (MHD) [7,11]:

$$
\left\{\begin{array}{l}
\partial_{t} \rho+\operatorname{div}(\rho u)=0, \\
\partial_{t}(\rho u)+\operatorname{div}(\rho u \otimes u)+\nabla p=\operatorname{curl} B \times B, \\
\partial_{t}(\rho e)+\operatorname{div}(\rho u e)+p \operatorname{div} u-\mu \Delta \theta=\kappa|\operatorname{curl} B|^{2}, \\
\partial_{t} B=\operatorname{curl} E, \quad E=u \times B-\kappa \operatorname{curl} B, \\
\operatorname{div} B=0,
\end{array}\right.
$$

where $\rho, u, \theta$ and $B$ denote the density, velocity, temperature and magnetic field, respectively, and $E$ is the electric field. The fluid is assumed to obey the ideal polytropic law, so the pressure $p=R \rho \theta$ and the internal energy $e=c_{v} \theta$ with constants $R, c_{v}>0 . \mu>0$ is the heat conduction coefficient and $\kappa>0$ is the magnetic diffusion coefficient.

The main difficulty of studying the global well-posedness of (1.1) lies in the absence of the viscosity. Similar to the Navier-Stokes equations, the viscous and resistive (incompressible and compressible) MHD system has a unique global classical solution, at least for the small initial data, see $[5,9,18]$ for instance. On the other hand, it is remarkable that the ideal incompressible homogeneous MHD system in the whole space also admits a unique global classical solution around a nonzero uniform magnetic field $[2,3,8,22]$. It is then natural to ask whether the MHD systems with only the viscosity or resistivity admit global classical solutions or develop singularities in finite time. The global existence of classical solutions to the viscous and non-resistive MHD systems has been established around a nonzero uniform magnetic field. For the Cauchy problem, we refer to $[1,13,15,25,27]$ for the incompressible homogeneous case and [24] for the 2D compressible isentropic case. For the initial boundary value problem, the global well-posedness has been proved only for the case of a horizontally flat strip domain, see [16] for the 2D incompressible homogeneous system around a uniform horizontal magnetic field and [20] for the 3D (incompressible and compressible) systems around a uniform non-horizontal magnetic field. The inviscid and resistive incompressible homogeneous 2D MHD system has a global weak solution in $H^{1}$, but the question whether such weak solutions are unique or can be improved to be global classical solutions remains open $[4,10,12]$. For a $2 \mathrm{D}$ periodic domain, [28] showed the global existence of classical solutions around a nonzero uniform magnetic field when the initial data has certain symmetries, and [23] proved a global well-posedness around the zero magnetic field.

In this paper, we consider the compressible MHD system (1.1) in the strip domain $\Omega=\mathbb{T}^{2} \times(0,1)$ for $\mathbb{T}=\mathbb{R} / \mathbb{Z}$, with the following boundary conditions: 


$$
u \cdot e_{3}=0, \quad \nabla \theta \cdot e_{3}=0, \quad B \cdot e_{3}=\bar{B} \cdot e_{3}, \quad E \times e_{3}=0 \quad \text { on } \partial \Omega,
$$

where $\bar{B}$ is the uniform magnetic field of the background. We will prove the global existence of classical solutions to (1.1)-(1.2) around the steady state $(\bar{\rho}, 0, \bar{\theta}, \bar{B})$ with the constant density $\bar{\rho}>0$ and temperature $\bar{\theta}>0$ and $\bar{B}_{3} \neq 0$.

Set

$$
\varrho=\rho-\bar{\rho}, \quad u=u, \quad \vartheta=\theta-\bar{\theta}, \quad b=B-\bar{B} .
$$

Then the problem under consideration can be reformulated as

$$
\begin{cases}\left(\partial_{t}+u \cdot \nabla\right) \varrho+\rho \operatorname{div} u=0 & \text { in } \Omega, \\ \rho\left(\partial_{t}+u \cdot \nabla\right) u+\nabla p=\operatorname{curl} b \times(\bar{B}+b) & \text { in } \Omega, \\ c_{v} \rho\left(\partial_{t}+u \cdot \nabla\right) \vartheta+p \operatorname{div} u-\mu \Delta \vartheta=\kappa|\operatorname{curl} b|^{2} & \text { in } \Omega, \\ \partial_{t} b=\operatorname{curl} E, E=u \times(\bar{B}+b)-\kappa \operatorname{curl} b & \text { in } \Omega, \\ \operatorname{div} b=0 & \text { in } \Omega, \\ u_{3}=0, \partial_{3} \vartheta=0, b_{3}=0, E \times e_{3}=0 & \text { on } \partial \Omega, \\ \left.(\varrho, u, \vartheta, b)\right|_{t=0}=\left(\varrho_{0}, u_{0}, \vartheta_{0}, b_{0}\right) . & \end{cases}
$$

Recall that the conditions $\operatorname{div} b=0$ in $\Omega$ and $b_{3}=0$ on $\partial \Omega$ in (1.4) should be regarded as the constraints on the initial data

$$
\operatorname{div} b_{0}=0 \text { in } \Omega \text { and } b_{0,3}=0 \text { on } \partial \Omega .
$$

Indeed, it follows from $\partial_{t} b=\operatorname{curl} E$ in $\Omega$ and $E \times e_{3}=0$ on $\partial \Omega$ in (1.4) that $\partial_{t} \operatorname{div} b=0$ in $\Omega$ and $\partial_{t} b_{3}=0$ on $\partial \Omega$.

Note the entropy-dissipation structure of (1.4): for a smooth solution, it holds that

$$
\begin{aligned}
& \frac{d}{d t} \int_{\Omega}\left(\frac{1}{2} \rho|u|^{2}+\rho c_{v}\left(\theta-\bar{\theta} \ln \frac{\theta}{\bar{\theta}}-\bar{\theta}\right)+R \bar{\theta}\left(\rho \ln \frac{\rho}{\bar{\rho}}-\rho+\bar{\rho}\right)+\frac{1}{2}|b|^{2}\right) \\
& \quad+\int_{\Omega}\left(\frac{\mu \bar{\theta}}{\theta^{2}}|\nabla \vartheta|^{2}+\frac{\kappa \bar{\theta}}{\theta}|\operatorname{curl} b|^{2}\right)=0 .
\end{aligned}
$$

Hereafter the differential elements $d x$ of the integrals over $\Omega$ are omitted. Furthermore, the following conservation laws hold:

$$
\frac{d}{d t} \int_{\Omega} \varrho=0, \quad \frac{d}{d t} \int_{\Omega}\left(\frac{1}{2} \rho|u|^{2}+c_{v} \rho \vartheta+\frac{1}{2}|b|^{2}\right)=0, \quad \frac{d}{d t} \int_{\Omega} b_{h}=0,
$$

where $b_{h}=\left(b_{1}, b_{2}\right)$, etc. It follows that if initially

$$
\int_{\Omega} \varrho_{0}=0, \quad \int_{\Omega}\left(\frac{1}{2} \rho_{0}\left|u_{0}\right|^{2}+c_{v} \rho_{0} \vartheta_{0}+\frac{1}{2}\left|b_{0}\right|^{2}\right)=0, \quad \int_{\Omega} b_{0, h}=0,
$$


then for any time $t>0$,

$$
\int_{\Omega} \varrho=0, \quad \int_{\Omega}\left(\frac{1}{2} \rho|u|^{2}+c_{v} \rho \vartheta+\frac{1}{2}|b|^{2}\right)=0, \quad \int_{\Omega} b_{h}=0 .
$$

(1.9) allows the use of the Poincare inequality for $\varrho, \vartheta, b_{h}$ in our analysis of the global well-posedness.

Let $H^{k}(\Omega), k \geq 0$ and $H^{s}\left(\mathbb{T}^{2}\right), s \in \mathbb{R}$ be the usual Sobolev spaces with norms denoted by $\|\cdot\|_{m}$ and $|\cdot|_{s}$, respectively. For an integer $N \geq 8$, define the high-order energy as

$$
\mathcal{E}_{2 N}:=\sum_{j=0}^{2 N}\left\|\partial_{t}^{j}(\varrho, u)\right\|_{2 N-j}^{2}+\sum_{j=0}^{2 N-1}\left\|\partial_{t}^{j}(\vartheta, b)\right\|_{2 N-j+1}^{2}+\left\|\partial_{t}^{2 N}(\vartheta, b)\right\|_{0}^{2} .
$$

The key part in proving the global well-posedness of (1.4) is to show that $\mathcal{E}_{2 N}(t)$ is bounded for all $t \geq 0$. To this end, we need to derive a sufficiently fast time-decay rate of certain lower-order Sobolev norms of the solution, which will follow from a set of energy-dissipation estimates. For $n=N+4, \ldots, 2 N$, define the dissipations as

$$
\mathfrak{D}_{n}:=\sum_{j=0}^{n-1}\left\|\partial_{t}^{j}(\varrho, u)\right\|_{n-j-1}^{2}+\sum_{j=0}^{n-2}\left\|\partial_{t}^{j}(\vartheta, b)\right\|_{n-j}^{2}+\sum_{j=0}^{n}\left\|\partial_{t}^{j}(\vartheta, b)\right\|_{1, n-j}^{2}
$$

and the corresponding energies as

$$
\begin{aligned}
\mathfrak{E}_{n}:= & \sum_{j=0}^{n}\left\|\partial_{t}^{j} \varrho\right\|_{n-j}^{2}+\|u\|_{0, n}^{2}+\|u\|_{n-1}^{2}+\sum_{j=1}^{n}\left\|\partial_{t}^{j} u\right\|_{n-j}^{2} \\
& +\|(\vartheta, b)\|_{n}^{2}+\sum_{j=1}^{n-1}\left\|\partial_{t}^{j}(\vartheta, b)\right\|_{n-j+1}^{2}+\left\|\partial_{t}^{n}(\vartheta, b)\right\|_{0}^{2} .
\end{aligned}
$$

Here the anisotropic Sobolev norm $\|\cdot\|_{m, \ell}$ is defined as

$$
\|f\|_{m, \ell}:=\sum_{\alpha \in \mathbb{N}^{2},|\alpha| \leq \ell}\left\|\partial^{\alpha} f\right\|_{m} .
$$

Now the main result of this paper is stated as follows.

Theorem 1.1. Assume that $\mu>0, \kappa>0$ and $\bar{\rho}>0, \bar{\theta}>0, \bar{B}_{3} \neq 0$. Let $N \geq 8$ be an integer. Assume that $\varrho_{0}, u_{0} \in H^{2 N}(\Omega)$ and $\vartheta_{0}, b_{0} \in H^{2 N+1}(\Omega)$ are given such that $\mathcal{E}_{2 N}(0)<+\infty$ and that the $2 N$-th order compatibility conditions required for the local well-posedness of (1.4)

$$
\partial_{t}^{j} u_{3}(0)=0, \quad \partial_{3} \partial_{t}^{j} \vartheta(0)=0, \quad \partial_{t}^{j} E(0) \times e_{3}=0 \quad \text { on } \partial \Omega \quad \text { for } \quad j=0, \ldots, 2 N-1,
$$


(1.5) and (1.8) are satisfied. There exists a universal constant $\varepsilon_{0}>0$ such that if $\mathcal{E}_{2 N}(0) \leq$ $\varepsilon_{0}$, then there exists a unique global solution $(\varrho, u, \vartheta, b)$ to $(1.4)$ satisfying that for all $t \geq 0$,

$$
\mathcal{E}_{2 N}(t) \lesssim \mathcal{E}_{2 N}(0)
$$

and

$$
\sum_{j=0}^{N-6}(1+t)^{N-5-j} \mathfrak{E}_{N+4+j}(t)+\sum_{j=0}^{N-6} \int_{0}^{t}(1+s)^{N-5-j} \mathfrak{D}_{N+4+j}(s) d s \lesssim \mathcal{E}_{2 N}(0) .
$$

Remark 1.1. Note that the global well-posedness of compressible inviscid heatconductive flows for the small initial data has been established only in 1D (see e.g., $[9,14,19,26])$, but the one in multi-D is unknown. So our result reveals the strong stabilizing effect of the transversal magnetic field and resistivity. In a forthcoming paper, we will use some ideas developed here to prove the global well-posedness of free interface problems for the compressible MHD system (1.1), which generalizes our previous work [21] of the inviscid and resistive incompressible homogeneous MHD system.

Remark 1.2. Our result here relies crucially on the consideration of the heatconductive flows, and it does not hold for isentropic flows or the case without heat conduction; for example, taking $B=\bar{B}=e_{3}$, these flows are reduced to the $1 \mathrm{D}$ compressible Euler flows for which in general one may expect the formation of shock in finite time. One of the key points here is that the heat conduction produces the dissipation control of the divergence of the velocity, as explained below.

Note that the local well-posedness of (1.4) (for $\rho, \theta>0$ ) for $\mathcal{E}_{2 N}<\infty$ with $N \geq 8$ is classical, which can be proved by using an iteration argument based on the solvability of the compressible Euler equations [17] and the parabolic problems for the temperature and magnetic field [6]. Hence, by a standard continuity argument, to prove the global well-posedness in Theorem 1.1 it suffices to derive a priori the estimate (1.15).

The basic strategy in our analysis is to use first the entropy-dissipation structure (1.6) to get the tangential energy evolution estimates of the solution to (1.4) as well as its temporal and horizontal spatial derivatives that preserve the boundary conditions, which will be carried out in Section 2. The next step is to derive the estimates involving normal derivatives by exploiting further the structures of the equations and boundary conditions, and the key here is to observe the following equations for the fluid vorticity curlu: applying curl to the second equation in 
(1.4), and then using the horizontal components of the fourth equation to replace $\partial_{3}^{2} b_{h}$ and the first equation to replace $\operatorname{div} u$, one finds

$$
\begin{aligned}
& \rho\left(\partial_{t}+u \cdot \nabla\right)(\operatorname{curl} u)_{i}+\frac{\bar{B}_{3}^{2}}{\kappa}(\operatorname{curl} u)_{i} \\
= & \bar{B}_{h} \cdot \nabla_{h}(\operatorname{curl} b)_{i}+(-1)^{i+1} \bar{B}_{3} \partial_{3} \partial_{3-i} b_{3}+(-1)^{i+1} \frac{\bar{B}_{3}}{\kappa}\left(\kappa \Delta_{h} b_{3-i}-\partial_{t} b_{3-i}\right) \\
& \quad+(-1)^{i+1} \frac{\bar{B}_{3}}{\kappa}\left(\bar{B}_{h} \cdot \nabla_{h} u_{3-i}+\bar{B}_{3} \partial_{3-i} u_{3}+\frac{\bar{B}_{3-i}}{\bar{\rho}} \partial_{t} \varrho\right)+\cdots, \quad i=1,2
\end{aligned}
$$

where $\nabla_{h}=\left(\partial_{1}, \partial_{2}\right), \Delta_{h}=\partial_{1}^{2}+\partial_{2}^{2}$ and “+ $+\cdots$ " means plus some nonlinear terms. As $\kappa>0$ and $\bar{B}_{3} \neq 0$, one thus sees that there is an induced damping effect for (curl $\left.u\right)_{h}$ in (1.17). By controlling the linear terms in the last line of (1.17) in terms of the tangential energy at the beginning, in a recursive way in terms of the number of normal derivatives one derives the following full energy estimates:

$$
\mathcal{E}_{2 N}(t) \lesssim \mathcal{E}_{2 N}(0)+\int_{0}^{t} \sqrt{\mathfrak{E}_{N+4}} \mathcal{E}_{2 N}
$$

Various elliptic estimates are involved in the derivation of (1.18), see Section 3 for the details.

Note that if $\sqrt{\mathfrak{E}_{N+4}}$ is integrable in time, then (1.18) can be closed to be (1.15). This will be achieved by using

$$
\frac{d}{d t} \mathfrak{E}_{n}+\mathfrak{D}_{n} \leq 0, \quad n=N+4, \ldots, 2 N-2 .
$$

(1.19) is derived in an elaborate way by controlling instead the linear terms in the last line of (1.17) in terms of the tangential dissipation. To achieve this, one notes first from the fourth equation in (1.4) that

$$
\partial_{t} b-\kappa \Delta b=\bar{B} \cdot \nabla u-\bar{B} \operatorname{div} u+\cdots .
$$

It is then crucial here to use the third equation in (1.4) to replace $\operatorname{div} u$ in (1.20) to find

$$
\partial_{t} b-\kappa \Delta b=\bar{B} \cdot \nabla u+\frac{\bar{B}}{\bar{p}}\left(c_{v} \bar{\rho} \partial_{t} \vartheta-\mu \Delta \vartheta\right)+\cdots
$$

As mentioned in Remark 1.2, it should be pointed out here that such replacement is effective only for $\mu>0$ since there is no priori tangential dissipation estimates of $\partial_{t} \vartheta$ if $\mu=0$, and the same reason holds also for that one could not use the first equation in (1.4) to replace $\operatorname{div} u$ by $\partial_{t} \varrho / \bar{\rho}$ in (1.20). Now using the vertical 
and horizontal components of (1.21), respectively, one then gets the tangential dissipation estimates of the following two quantities:

$$
\bar{B} \cdot \nabla\left(u_{3}-\frac{\mu}{\bar{p}} \partial_{3} \vartheta\right), \quad \bar{B} \cdot \nabla\left(\kappa \partial_{3} b_{h}+\bar{B}_{3} u_{h}-\frac{\mu \bar{B}_{h}}{\bar{p}} \partial_{3} \vartheta\right)
$$

Thus one can use the Poincaré-type inequalities related to $\bar{B} \cdot \nabla$ for $\bar{B}_{3} \neq 0$ together with the boundary conditions on $\partial \Omega$ to derive the tangential dissipation estimates of $u$. The tangential dissipation estimates of $\partial_{t} \varrho$ then follow from the interaction between the first and second equations in (1.4).

Now observe that $\mathfrak{E}_{\ell} \leq \mathfrak{D}_{\ell+1}$. Then by employing a time weighted inductive argument based on (1.19), one concludes (1.16), which implies in particular a decay of $\sqrt{\mathfrak{E}_{N+4}}$ with the rate $(1+t)^{-(N-5) / 2}$, see Section 4 for more details. Consequently, this scheme of the a priori estimates is closed by requiring $N \geq 8$.

Notation. Now we list the conventions for notation in this paper. $C>0$ denotes generic constants independent of the data and time, but may depend on the parameters of the problem, $\kappa, \mu, \bar{B}, \bar{\rho}, \bar{\theta}$ and $N$, which is referred to as "universal" and allowed to change from line to line. $A_{1} \lesssim A_{2}$ means that $A_{1} \leq C A_{2}$ for a universal constant $C>0, A_{1} \lesssim A_{2}+A_{3}$ means that $A_{1} \leq A_{2}+C A_{3}$ and

$$
\partial_{t} A_{1}+A_{2} \lesssim A_{3} \quad \text { means } \quad \partial_{t} \widetilde{A}_{1}+A_{2} \lesssim A_{3} \quad \text { for } \quad A_{1} \lesssim \widetilde{A}_{1} \lesssim A_{1}
$$

$\mathbb{N}=\{0,1, \ldots\}$ denotes for the collection of non-negative integers. When using space-time differential multi-indices, we write $\mathbb{N}^{1+d}=\left\{\alpha=\left(\alpha_{0}, \alpha_{1}, \ldots, \alpha_{d}\right)\right\}$ to emphasize that the 0 -index term is related to temporal derivatives. For just spatial derivatives, we write $\mathbb{N}^{d}$. For $\alpha \in \mathbb{N}^{1+d}, \partial^{\alpha}=\partial_{t}^{\alpha_{0}} \partial_{1}^{\alpha_{1}} \ldots \partial_{d}^{\alpha_{d}}$. We define the standard commutators

$$
\left[\partial^{\alpha}, f\right] g=\partial^{\alpha}(f g)-f \partial^{\alpha} g \quad \text { and } \quad\left[\partial^{\alpha}, f, g\right]=\partial^{\alpha}(f g)-f \partial^{\alpha} g-\partial^{\alpha} f g
$$

\section{Tangential energy evolution}

In this section, we will derive energy evolution estimates for temporal and horizontal spatial derivatives of the solution to (1.4) by using the entropy-dissipation structure (1.6). It will be assumed throughout the rest of the paper that the solution to (1.4) is given on $[0, T]$ and obeys the a priori assumption

$$
\mathcal{E}_{2 N}(t) \leq \delta, \quad \forall t \in[0, T]
$$


for a sufficiently small constant $\delta>0$. This implies in particular that

$$
\frac{1}{2} \bar{\rho} \leq \rho \leq 2 \bar{\rho}, \quad \frac{1}{2} \bar{\theta} \leq \theta \leq 2 \bar{\theta}, \quad \forall(t, x) \in[0, T] \times \bar{\Omega} .
$$

Note that (2.1) and (2.2) will be always used in the following; in particular, the smallness (2.1) is used in many nonlinear estimates so that various polynomials of $\mathcal{E}_{2 N}$ are bounded by $C \mathcal{E}_{2 N}$.

In order to use the nonlinear structure of (1.4) to derive the tangential energy evolution estimates at the $2 N$ level, one applies $\partial^{\alpha}$ for $\alpha \in \mathbb{N}^{1+2}$ to (1.4) to find

$$
\begin{cases}\left(\partial_{t}+u \cdot \nabla\right) \partial^{\alpha} \varrho+\rho \operatorname{div} \partial^{\alpha} u=F^{1, \alpha} & \text { in } \Omega, \\ \rho\left(\partial_{t}+u \cdot \nabla\right) \partial^{\alpha} u+\nabla\left(R \theta \partial^{\alpha} \varrho+R \rho \partial^{\alpha} \vartheta\right)=\operatorname{curl} \partial^{\alpha} b \times(\bar{B}+b)+F^{2, \alpha} & \text { in } \Omega, \\ c_{v} \rho\left(\partial_{t}+u \cdot \nabla\right) \partial^{\alpha} \vartheta+p \operatorname{div} \partial^{\alpha} u-\mu \Delta \partial^{\alpha} \vartheta=F^{3, \alpha} & \text { in } \Omega, \\ \partial_{t} \partial^{\alpha} b=\operatorname{curl} \partial^{\alpha} E, \partial^{\alpha} E=\partial^{\alpha} u \times(\bar{B}+b)-\kappa \operatorname{curl} \partial^{\alpha} b+F^{4, \alpha} & \text { in } \Omega, \\ \operatorname{div} \partial^{\alpha} b=0 & \text { in } \Omega, \\ \partial^{\alpha} u_{3}=0, \partial_{3} \partial^{\alpha} \vartheta=0, \partial^{\alpha} b_{3}=0, \partial^{\alpha} E \times e_{3}=0 & \text { on } \partial \Omega,\end{cases}
$$

where

$$
\begin{aligned}
& F^{1, \alpha}=-\left[\partial^{\alpha}, u\right] \cdot \nabla \varrho-\left[\partial^{\alpha}, \varrho\right] \operatorname{div} u, \\
& F^{2, \alpha}=-\left[\partial^{\alpha}, \rho\left(\partial_{t}+u \cdot \nabla\right)\right] u-\nabla\left(R\left[\partial^{\alpha}, \varrho, \vartheta\right]\right)-\left[\partial^{\alpha}, b\right] \times \operatorname{curl} b, \\
& F^{3, \alpha}=-\left[\partial^{\alpha}, c_{v} \rho\left(\partial_{t}+u \cdot \nabla\right)\right] \vartheta-\left[\partial^{\alpha}, p\right] \operatorname{div} u+\kappa \partial^{\alpha}\left(|\operatorname{curl} b|^{2}\right), \\
& F^{4, \alpha}=-\left[\partial^{\alpha}, b\right] \times u
\end{aligned}
$$

with notation of commutators given in (1.24).

These nonlinear terms $F^{i, \alpha}$ are estimated as follows.

Lemma 2.1. For $|\alpha| \leq 2 N$, it holds that

$$
\left\|\left(F^{1, \alpha}, F^{2, \alpha}, F^{3, \alpha}, F^{4, \alpha}\right)\right\|_{0}^{2} \lesssim \mathfrak{E}_{N+4} \mathcal{E}_{2 N} .
$$

Proof. Note that all terms in $F^{i, \alpha}$ are at least quadratic and each term can be written in the form $X Y$, where $X$ involves fewer derivatives than $Y$. One may use the definition of $\mathcal{E}_{n}$ to estimate $\|Y\|_{0}^{2} \lesssim \mathcal{E}_{2 N}$ and the usual Sobolev embeddings along with the definition of $\mathfrak{E}_{n}$ to estimate $\|X\|_{L^{\infty}}^{2} \lesssim \mathfrak{E}_{N+4}$. Then

$$
\|X Y\|_{0}^{2} \leq\|X\|_{L^{\infty}}^{2}\|Y\|_{0}^{2} \lesssim \mathfrak{E}_{N+4} \mathcal{E}_{2 N}
$$

and the estimate (2.5) follows by summing terms. 
For $n \geq 3$, define the tangential energy by

$$
\overline{\mathcal{E}}_{n}:=\sum_{j=0}^{n}\left\|\partial_{t}^{j}(\varrho, u, \vartheta, b)\right\|_{0, n-j}^{2}
$$

and the corresponding dissipation by

$$
\overline{\mathcal{D}}_{n}:=\sum_{j=0}^{n}\left\|\partial_{t}^{j}(\vartheta, b)\right\|_{1, n-j^{\prime}}^{2}
$$

where anisotropic Sobolev norms (1.13) have been used. Then one has the following tangential energy evolution at the $2 \mathrm{~N}$ level.

Proposition 2.1. It holds that

$$
\frac{d}{d t} \overline{\mathcal{E}}_{2 N}+\overline{\mathcal{D}}_{2 N} \lesssim \sqrt{\mathfrak{E}_{N+4}} \mathcal{E}_{2 N}
$$

Proof. Let $\alpha \in \mathbb{N}^{1+2}$ with $|\alpha| \leq 2 N$. First, taking the inner product of the second equation in (2.3) with $\partial^{\alpha} u$ and then integrating by parts over $\Omega$, by using the first equation in (1.4) and the boundary conditions, one has

$$
\begin{aligned}
& \frac{1}{2} \frac{d}{d t} \int_{\Omega} \rho\left|\partial^{\alpha} u\right|^{2}-\int_{\Omega}\left(R \theta \partial^{\alpha} \varrho+R \rho \partial^{\alpha} \vartheta\right) \operatorname{div} \partial^{\alpha} u \\
= & \int_{\Omega} \operatorname{curl} \partial^{\alpha} b \times(\bar{B}+b) \cdot \partial^{\alpha} u+\int_{\Omega} F^{2, \alpha} \cdot \partial^{\alpha} u .
\end{aligned}
$$

The first equation in (2.3) leads to

$$
\begin{aligned}
& -\int_{\Omega} R \theta \partial^{\alpha} \varrho \operatorname{div} \partial^{\alpha} u=\int_{\Omega} \frac{R \theta}{\rho}\left(\partial_{t} \partial^{\alpha} \varrho+u \cdot \nabla \partial^{\alpha} \varrho-F^{1, \alpha}\right) \partial^{\alpha} \varrho \\
= & \frac{1}{2} \frac{d}{d t} \int_{\Omega} \frac{R \theta}{\rho}\left|\partial^{\alpha} \varrho\right|^{2}-\frac{1}{2} \int_{\Omega} R \rho\left(\partial_{t}+u \cdot \nabla\right)\left(\frac{\theta}{\rho^{2}}\right)\left|\partial^{\alpha} \varrho\right|^{2}-\int_{\Omega} \frac{R \theta}{\rho} F^{1, \alpha} \partial^{\alpha} \varrho .
\end{aligned}
$$

Next, taking the inner product of the third equation with $\partial^{\alpha} \vartheta / \theta$ and using the relation $p=R \rho \theta$ give

$$
\begin{aligned}
& \frac{1}{2} \frac{d}{d t} \int_{\Omega} \frac{c_{v} \rho}{\theta}\left|\partial^{\alpha} \vartheta\right|^{2}+\int_{\Omega} \frac{\mu}{\theta}\left|\nabla \partial^{\alpha} \vartheta\right|^{2}+\int_{\Omega} R \rho \operatorname{div} \partial^{\alpha} u \partial^{\alpha} \vartheta \\
= & \frac{1}{2} \int_{\Omega} c_{v} \rho\left(\partial_{t}+u \cdot \nabla\right)\left(\frac{1}{\theta}\right)\left|\partial^{\alpha} \vartheta\right|^{2}-\int_{\Omega} \mu \partial^{\alpha} \vartheta \nabla\left(\frac{1}{\theta}\right) \cdot \nabla \partial^{\alpha} \vartheta+\int_{\Omega} F^{3, \alpha} \partial^{\alpha} \vartheta,
\end{aligned}
$$


while taking the inner product of the fourth equation with $\partial^{\alpha} b$ yields

$$
\begin{aligned}
& \frac{1}{2} \frac{d}{d t} \int_{\Omega}\left|\partial^{\alpha} b\right|^{2}+\kappa \int_{\Omega}\left|\operatorname{curl} \partial^{\alpha} b\right|^{2} \\
= & \int_{\Omega} \partial^{\alpha} u \times(\bar{B}+b) \cdot \operatorname{curl} \partial^{\alpha} b+\int_{\Omega} F^{4, \alpha} \cdot \operatorname{curl} \partial^{\alpha} b .
\end{aligned}
$$

Consequently, combining (2.9)-(2.12) yields that, by (2.5),

$$
\begin{aligned}
& \frac{1}{2} \frac{d}{d t} \int_{\Omega}\left(\frac{R \theta}{\rho}\left|\partial^{\alpha} \varrho\right|^{2}+\rho\left|\partial^{\alpha} u\right|^{2}+\frac{c_{v} \rho}{\theta}\left|\partial^{\alpha} \vartheta\right|^{2}+\left|\partial^{\alpha} b\right|^{2}\right) \\
& \quad+\int_{\Omega}\left(\frac{\mu}{\theta}\left|\nabla \partial^{\alpha} \vartheta\right|^{2}+\kappa\left|\operatorname{curl} \partial^{\alpha} b\right|^{2}\right) \\
& =\int_{\Omega}\left(\frac{1}{2} R \rho\left(\partial_{t}+u \cdot \nabla\right)\left(\frac{\theta}{\rho^{2}}\right)\left|\partial^{\alpha} \varrho\right|^{2}+\frac{1}{2} c_{v} \rho\left(\partial_{t}+u \cdot \nabla\right)\left(\frac{1}{\theta}\right)\left|\partial^{\alpha} \vartheta\right|^{2}\right. \\
& \left.\quad-\mu \partial^{\alpha} \vartheta \nabla\left(\frac{1}{\theta}\right) \cdot \nabla \partial^{\alpha} \vartheta\right) \\
& \quad+\int_{\Omega}\left(\frac{R \theta}{\rho} F^{1, \alpha} \partial^{\alpha} \varrho+F^{2, \alpha} \cdot \partial^{\alpha} u+F^{3, \alpha} \partial^{\alpha} \vartheta+F^{4, \alpha} \cdot \operatorname{curl} \partial^{\alpha} b\right) \\
& \lesssim \sqrt{\mathfrak{E}_{N+4}}\left(\left\|\partial^{\alpha} \varrho\right\|_{0}^{2}+\left\|\partial^{\alpha} \vartheta\right\|_{0}^{2}+\left\|\partial^{\alpha} \vartheta\right\|_{0}\left\|\nabla \partial^{\alpha} \vartheta\right\|_{0}\right)+\left\|F^{1, \alpha}\right\|_{0}\left\|\partial^{\alpha} \varrho\right\|_{0} \\
& \quad+\left\|F^{2, \alpha}\right\|_{0}\left\|\partial^{\alpha} u\right\|_{0}+\left\|F^{3, \alpha}\right\|_{0}\left\|\partial^{\alpha} \vartheta\right\|_{0}+\left\|F^{4, \alpha}\right\|_{0}\left\|\operatorname{curl} \partial^{\alpha} b\right\|_{0} \\
& \lesssim \sqrt{\mathfrak{E}_{N+4}} \mathcal{E}_{2 N}+\sqrt{\mathfrak{E}_{N+4}} \sqrt{\mathcal{E}_{2 N}}\left(\left\|\nabla \partial^{\alpha} \vartheta\right\|_{0}+\left\|\operatorname{curl} \partial^{\alpha} b\right\|_{0}\right) .
\end{aligned}
$$

By Cauchy's inequality, one deduces from (2.13) that, by the convention notation (1.23),

$$
\frac{d}{d t}\left\|\partial^{\alpha}(\varrho, u, \vartheta, b)\right\|_{0}^{2}+\left\|\nabla \partial^{\alpha} \vartheta\right\|_{0}^{2}+\left\|\operatorname{curl} \partial^{\alpha} b\right\|_{0}^{2} \lesssim \sqrt{\mathfrak{E}_{N+4}} \mathcal{E}_{2 N}
$$

It follows by using the Poincaré inequality and (1.9) that

$$
\left\|\partial^{\alpha} \vartheta\right\|_{0}^{2} \lesssim\left\|\nabla \partial^{\alpha} \vartheta\right\|_{0}^{2}+\left|\int_{\Omega} \partial^{\alpha} \vartheta\right|^{2} \lesssim\left\|\nabla \partial^{\alpha} \vartheta\right\|_{0}^{2}+\mathfrak{E}_{N+4} \mathcal{E}_{2 N}
$$

Employing the Hodge-type estimates (A.3) of Lemma A.1 with $r=1$ and by (1.9), one finds

$$
\left\|\partial^{\alpha} b\right\|_{1}^{2} \lesssim\left\|\operatorname{curl} \partial^{\alpha} b\right\|_{0}^{2}
$$

Then the estimate (2.8) follows from (2.14) by summing over such $\alpha$. 
Now we rewrite (1.4) as a perturbation of the linearized equations

$$
\begin{cases}\partial_{t} \varrho+\bar{\rho} \operatorname{div} u=G^{1} & \text { in } \Omega, \\ \bar{\rho} \partial_{t} u+\nabla(R \bar{\theta} \varrho+R \bar{\rho} \vartheta)=\operatorname{curl} b \times \bar{B}+G^{2} & \text { in } \Omega, \\ c_{v} \bar{\rho} \partial_{t} \vartheta+\bar{p} \operatorname{div} u-\mu \Delta \vartheta=G^{3} & \text { in } \Omega, \\ \partial_{t} b-\kappa \Delta b=\bar{B} \cdot \nabla u-\bar{B} \operatorname{div} u+G^{4} & \text { in } \Omega, \\ \operatorname{div} b=0 & \text { in } \Omega, \\ u_{3}=0, \partial_{3} \vartheta=0, \quad b_{3}=0, \kappa \partial_{3} b_{h}=-\bar{B}_{3} u_{h} & \text { on } \partial \Omega,\end{cases}
$$

where $\bar{p}=R \bar{\rho} \bar{\theta}$ and

$$
\begin{aligned}
& G^{1}=-\operatorname{div}(\varrho u), \\
& G^{2}=-\varrho \partial_{t} u-\rho u \cdot \nabla u-\nabla(\operatorname{R} \varrho \vartheta)+\operatorname{curl} b \times b, \\
& G^{3}=-c_{v} \varrho \partial_{t} \vartheta-c_{v} \rho u \cdot \nabla \vartheta-(p-\bar{p}) \operatorname{div} u+\kappa|\operatorname{curl} b|^{2}, \\
& G^{4}=\operatorname{curl}(u \times b) .
\end{aligned}
$$

The nonlinear terms $G^{i}$ are estimated as follows.

Lemma 2.2. It holds that

$$
\sum_{j=0}^{2 N-1}\left\|\partial_{t}^{j}\left(G^{1}, G^{2}, G^{3}, G^{4}\right)\right\|_{2 N-j-1}^{2} \lesssim \min \left\{\mathfrak{E}_{N+4}, \mathfrak{D}_{N+4}\right\} \mathcal{E}_{2 N}
$$

Proof. (2.19) can be proved similarly to Lemma 2.1.

One has the following tangential energy evolution at the $N+4, \ldots, 2 N-2$ levels.

Proposition 2.2. For $n=N+4, \ldots, 2 N-2$, it holds that

$$
\frac{d}{d t}\left(\overline{\mathcal{E}}_{n}-\overline{\mathcal{B}}_{n}\right)+\overline{\mathcal{D}}_{n} \lesssim \sqrt{\mathcal{E}_{2 N}} \mathfrak{D}_{n}
$$

where $\overline{\mathcal{B}}_{n}$ is defined by (2.27), which satisfies

$$
\left|\overline{\mathcal{B}}_{n}\right| \lesssim \sqrt{\mathcal{E}_{2 N}} \mathfrak{E}_{n}
$$

Proof. For $n=N+4, \ldots, 2 N-2$, similarly as the derivation of (2.13), one deduces from (2.17) that for $\alpha \in \mathbb{N}^{1+2}$ with $|\alpha| \leq n$,

$$
\frac{1}{2} \frac{d}{d t} \int_{\Omega}\left(\frac{R \bar{\theta}}{\bar{\rho}}\left|\partial^{\alpha} \varrho\right|^{2}+\bar{\rho}\left|\partial^{\alpha} u\right|^{2}+\frac{c_{v} \bar{\rho}}{\bar{\theta}}\left|\partial^{\alpha} \vartheta\right|^{2}+\left|\partial^{\alpha} b\right|^{2}\right)
$$




$$
\begin{gathered}
+\int_{\Omega}\left(\frac{\mu}{\bar{\theta}}\left|\nabla \partial^{\alpha} \vartheta\right|^{2}+\kappa\left|\operatorname{curl} \partial^{\alpha} b\right|^{2}\right) \\
=\int_{\Omega}\left(\frac{R \bar{\theta}}{\bar{\rho}} \partial^{\alpha} G^{1} \partial^{\alpha} \varrho+\partial^{\alpha} G^{2} \cdot \partial^{\alpha} u+\partial^{\alpha} G^{3} \partial^{\alpha} \vartheta+\partial^{\alpha} G^{4} \cdot \operatorname{curl} \partial^{\alpha} b\right) .
\end{gathered}
$$

It follows from (2.19) that

$$
\begin{aligned}
& \int_{\Omega}\left(\partial^{\alpha} G^{3} \partial^{\alpha} \vartheta+\partial^{\alpha} G^{4} \cdot \operatorname{curl} \partial^{\alpha} b\right) \\
\lesssim & \left\|\partial^{\alpha} G^{3}\right\|_{0}\left\|\partial^{\alpha} \vartheta\right\|_{0}+\left\|\partial^{\alpha} G^{4}\right\|_{0}\left\|\operatorname{curl} \partial^{\alpha} b\right\|_{0} \lesssim \sqrt{\mathfrak{D}_{N+4} \mathcal{E}_{2 N}} \sqrt{\mathfrak{D}_{n}}
\end{aligned}
$$

Now we estimate the term $G^{1}$. If $|\alpha| \leq n-1$, then (2.19) implies

$$
\int_{\Omega} \partial^{\alpha} \varrho \partial^{\alpha} G^{1} \lesssim\left\|\partial^{\alpha} \varrho\right\|_{0}\left\|\partial^{\alpha} G^{1}\right\|_{0} \lesssim \sqrt{\mathfrak{D}_{n}} \sqrt{\mathfrak{D}_{N+4} \mathcal{E}_{2 N}}
$$

If $|\alpha|=n$ and $\alpha_{1}+\alpha_{2} \geq 1$, then one writes $\alpha=\alpha^{\prime}+\left(\alpha-\alpha^{\prime}\right)$ for $\alpha^{\prime} \in \mathbb{N}^{2}$ with $\alpha^{\prime} \leq \alpha$ and $\left|\alpha^{\prime}\right|=1$ and then integrates by parts over $\Omega$ to have, since $n \leq 4 N-2$ and by (2.19),

$$
\begin{gathered}
\int_{\Omega} \partial^{\alpha} \varrho \partial^{\alpha} G^{1}=\int_{\Omega} \partial^{\alpha-\alpha^{\prime}} \varrho \partial^{\alpha+\alpha^{\prime}} G^{1} \\
\lesssim\left\|\partial^{\alpha-\alpha^{\prime}} \varrho\right\|_{0}\left\|\partial^{\alpha+\alpha^{\prime}} G^{1}\right\|_{0} \lesssim \sqrt{\mathfrak{D}_{n}} \sqrt{\mathfrak{D}_{N+4} \mathcal{E}_{2 N}} .
\end{gathered}
$$

The remaining case, $\alpha_{0}=n$, can be handled by the integration by parts in $t$ and using (2.19) as

$$
\begin{aligned}
\int_{\Omega} \partial_{t}^{n} \varrho \partial_{t}^{n} G^{1} & =\frac{d}{d t} \int_{\Omega} \partial_{t}^{n-1} \varrho \partial_{t}^{n} G^{1}-\int_{\Omega} \partial_{t}^{n-1} \varrho \partial_{t}^{n+1} G^{1} \\
& \lesssim \frac{d}{d t} \int_{\Omega} \partial_{t}^{n-1} \varrho \partial_{t}^{n} G^{1}+\left\|\partial_{t}^{n-1} \varrho\right\|_{0}\left\|\partial_{t}^{n+1} G^{1}\right\|_{0} \\
& \lesssim \frac{d}{d t} \int_{\Omega} \partial_{t}^{n-1} \varrho \partial_{t}^{n} G^{1}+\sqrt{\mathfrak{D}_{n}} \sqrt{\mathfrak{D}_{N+4} \mathcal{E}_{2 N}}
\end{aligned}
$$

Consequently, combining (2.24)-(2.26) (and doing the similar computations for the $G^{2}$ term) and (2.23), one concludes (2.20) from (2.22) by summing over such $\alpha$, where

$$
\overline{\mathcal{B}}_{n}:=\int_{\Omega}\left(\frac{R \bar{\theta}}{\bar{\rho}} \partial_{t}^{n-1} \varrho \partial_{t}^{n} G^{1}+\partial_{t}^{n-1} u \cdot \partial_{t}^{n} G^{2}\right),
$$

which, by (2.19), satisfies (2.21). 


\section{Boundedness estimates of $\mathcal{E}_{N}$}

In this section, we will explore further the structures of (1.4) to derive the estimates involving the normal derivatives of the solution, with the tangential energy evolution estimates in hand.

For the estimates of the normal derivatives of the velocity $u$, as for the Euler equations, a natural way is to estimate first the fluid vorticity, curl $u$, and then to use the Hodge-type estimates. Applying curl to the second equation in (1.4) yields that

$$
\rho\left(\partial_{t}+u \cdot \nabla\right) \operatorname{curl} u=\bar{B} \cdot \nabla \operatorname{curl} b+\operatorname{curl}(\operatorname{curl} b \times b)-\left[\operatorname{curl}, \rho\left(\partial_{t}+u \cdot \nabla\right)\right] u .
$$

It follows from the second component of the fourth equation in (2.17) that

$$
\begin{aligned}
& \bar{B} \cdot \nabla(\operatorname{curl} b)_{1} \equiv \bar{B}_{h} \cdot \nabla_{h}(\operatorname{curl} b)_{1}+\bar{B}_{3} \partial_{3}(\operatorname{curl} b)_{1} \\
= & \bar{B}_{h} \cdot \nabla_{h}(\operatorname{curl} b)_{1}+\bar{B}_{3} \partial_{3} \partial_{2} b_{3}+\frac{\bar{B}_{3}}{\kappa}\left(\kappa \Delta_{h} b_{2}-\partial_{t} b_{2}+\bar{B} \cdot \nabla u_{2}-\bar{B}_{2} \operatorname{div} u+G_{2}^{4}\right) .
\end{aligned}
$$

On the other hand, one can write, by using the first equation in (2.17), that

$$
\bar{B} \cdot \nabla u_{2}-\bar{B}_{2} \operatorname{div} u=\bar{B}_{h} \cdot \nabla_{h} u_{2}-\bar{B}_{3}(\operatorname{curl} u)_{1}+\bar{B}_{3} \partial_{2} u_{3}+\frac{\bar{B}_{2}}{\bar{\rho}}\left(\partial_{t} \varrho-G^{1}\right) .
$$

Hence, as a consequence of (3.2) and (3.3), the first component of (3.1) can be rewritten as

$$
\begin{aligned}
& \rho\left(\partial_{t}+u \cdot \nabla\right)(\operatorname{curl} u)_{1}+\frac{\bar{B}_{3}^{2}}{\kappa}(\operatorname{curl} u)_{1} \\
= & \bar{B}_{h} \cdot \nabla_{h}(\operatorname{curl} b)_{1}+\bar{B}_{3} \partial_{3} \partial_{2} b_{3}+\frac{\bar{B}_{3}}{\kappa}\left(\kappa \Delta_{h} b_{2}-\partial_{t} b_{2}+\bar{B}_{h} \cdot \nabla_{h} u_{2}+\bar{B}_{3} \partial_{2} u_{3}+\frac{\bar{B}_{2}}{\bar{\rho}} \partial_{t} \varrho\right) \\
& +\frac{\bar{B}_{3}}{\kappa}\left(-\frac{\bar{B}_{2}}{\bar{\rho}} G^{1}+G_{2}^{4}\right)+(\operatorname{curl}(\operatorname{curl} b \times b))_{1}-\left[\operatorname{curl}, \rho\left(\partial_{t}+u \cdot \nabla\right)\right] u_{1} \cdot
\end{aligned}
$$

Similarly, one has

$$
\begin{aligned}
& \rho\left(\partial_{t}+u \cdot \nabla\right)(\operatorname{curl} u)_{2}+\frac{\bar{B}_{3}^{2}}{\kappa}(\operatorname{curl} u)_{2} \\
= & \bar{B}_{h} \cdot \nabla_{h}(\operatorname{curl} b)_{2}-\bar{B}_{3} \partial_{3} \partial_{1} b_{3}-\frac{\bar{B}_{3}}{\kappa}\left(\kappa \Delta_{h} b_{1}-\partial_{t} b_{1}+\bar{B}_{h} \cdot \nabla_{h} u_{1}+\bar{B}_{3} \partial_{1} u_{3}+\frac{\bar{B}_{1}}{\bar{\rho}} \partial_{t} \varrho\right) \\
& -\frac{\bar{B}_{3}}{\kappa}\left(-\frac{\bar{B}_{1}}{\bar{\rho}} G^{1}+G_{1}^{4}\right)+(\operatorname{curl}(\operatorname{curl} b \times b))_{2}-\left[\operatorname{curl}, \rho\left(\partial_{t}+u \cdot \nabla\right)\right] u_{2} \cdot
\end{aligned}
$$


Eqs. (3.4) and (3.5) yield a transport-damping evolution structure for $(\operatorname{curl} u)_{h}$, which depends crucially on the positivity of the magnetic diffusion coefficient $\kappa>0$ and the non-vanishing of $\bar{B}_{3} \neq 0$.

Applying $\partial^{\alpha}$ for $\alpha \in \mathbb{N}^{1+3}$ with $|\alpha| \geq 1$ to (3.4) and (3.5) gives

$$
\rho\left(\partial_{t}+u \cdot \nabla\right) \partial^{\alpha}(\operatorname{curl} u)_{h}+\frac{\bar{B}_{3}^{2}}{\kappa} \partial^{\alpha}(\operatorname{curl} u)_{h}=\partial^{\alpha} L_{h}+\Phi_{h^{\prime}}^{\alpha}
$$

where for $i=1,2$,

$$
\begin{aligned}
L_{i}= & \bar{B}_{h} \cdot \nabla_{h}(\operatorname{curl} b)_{i}+(-1)^{i+1} \bar{B}_{3} \partial_{3} \partial_{3-i} b_{3} \\
& +(-1)^{i+1} \frac{\bar{B}_{3}}{\kappa}\left(\kappa \Delta_{h} b_{3-i}-\partial_{t} b_{3-i}+\bar{B}_{h} \cdot \nabla_{h} u_{3-i}+\bar{B}_{3} \partial_{3-i} u_{3}+\frac{\bar{B}_{3-i}}{\bar{\rho}} \partial_{t} \varrho\right)
\end{aligned}
$$

and

$$
\Phi_{h}^{\alpha}=\partial^{\alpha} \Phi_{h}-\left[\partial^{\alpha}, \rho\left(\partial_{t}+u \cdot \nabla\right)\right](\operatorname{curl} u)_{h}
$$

with that for $i=1,2$,

$$
\begin{aligned}
\Phi_{i}= & (-1)^{i+1} \frac{\bar{B}_{3}}{\kappa}\left(-\frac{\bar{B}_{3-i}}{\bar{\rho}} G^{1}+G_{3-i}^{4}\right)+(\operatorname{curl}(\operatorname{curl} b \times b))_{i} \\
& -\left[\operatorname{curl}, \rho\left(\partial_{t}+u \cdot \nabla\right)\right] u_{i} .
\end{aligned}
$$

The nonlinear terms $\Phi_{h}^{\alpha}$ can be estimated as follows.

Lemma 3.1. It holds that for $|\alpha| \leq 2 N-1$,

$$
\left\|\Phi_{h}^{\alpha}\right\|_{0}^{2} \lesssim \min \left\{\mathfrak{E}_{N+4}, \mathfrak{D}_{N+4}\right\} \mathcal{E}_{2 N} .
$$

Proof. The proof follows in the same way as for Lemmas 2.1. lows.

The estimates involving normal derivatives at the $2 \mathrm{~N}$ level are derived as fol-

Proposition 3.1. It holds that

$$
\begin{aligned}
& \frac{d}{d t}\left\|(\operatorname{curl} u)_{h}\right\|_{2 N-1}^{2}+\sum_{j=0}^{2 N}\left\|\partial_{t}^{j}(\varrho, u)\right\|_{2 N-j}^{2}+\sum_{j=0}^{2 N}\left\|\partial_{t}^{j}(\vartheta, b)\right\|_{2 N-j+1}^{2} \\
\lesssim & \overline{\mathcal{E}}_{2 N}+\overline{\mathcal{D}}_{2 N}+\mathfrak{E}_{N+4} \mathcal{E}_{2 N}
\end{aligned}
$$

and that

$$
\mathcal{E}_{2 N} \lesssim \overline{\mathcal{E}}_{2 N}+\left\|(\operatorname{curl} u)_{h}\right\|_{2 N-1}^{2}+\mathfrak{E}_{N+4} \mathcal{E}_{2 N}
$$


Proof. Fix $\ell=0, \ldots, 2 N-1$. Let $\alpha \in \mathbb{N}^{3}$ with $|\alpha| \leq 2 N-1$ such that $\alpha_{3} \leq 2 N-1-\ell$. Taking the inner product of (3.6) with $\partial^{\alpha}(\operatorname{curl} u)_{h}$ and then integrating by parts over $\Omega$, one obtains

$$
\begin{aligned}
& \frac{1}{2} \frac{d}{d t} \int_{\Omega} \rho\left|\partial^{\alpha}(\operatorname{curl} u)_{h}\right|^{2}+\frac{\bar{B}_{3}^{2}}{\kappa} \int_{\Omega}\left|\partial^{\alpha}(\operatorname{curl} u)_{h}\right|^{2} \\
= & \int_{\Omega}\left(\partial^{\alpha} L_{h}+\Phi_{h}^{\alpha}\right) \cdot \partial^{\alpha}(\operatorname{curl} u)_{h} .
\end{aligned}
$$

By Cauchy's inequality, (3.7) and (3.10), it follows from summing (3.13) over such $\alpha$ that

$$
\begin{aligned}
& \frac{d}{d t}\left\|(\operatorname{curl} u)_{h}\right\|_{2 N-1-\ell, \ell}^{2}+\left\|(\operatorname{curl} u)_{h}\right\|_{2 N-1-\ell, \ell}^{2} \lesssim \sum_{\alpha}\left\|\partial^{\alpha} L_{h}\right\|_{0}^{2}+\left\|\Phi_{h}^{\alpha}\right\|_{0}^{2} \\
\lesssim & \|u\|_{2 N-1-\ell, \ell+1}^{2}+\|b\|_{2 N-\ell, \ell+1}^{2}+\left\|\partial_{t}(b, \varrho)\right\|_{2 N-1}^{2}+\mathfrak{E}_{N+4} \mathcal{E}_{2 N} .
\end{aligned}
$$

Now employing the Hodge-type estimates (A.1) of Lemma A.1 with $r=2 N-\ell \geq 1$ and using the first equation in (2.17) and (2.19), one obtains

$$
\begin{aligned}
\|u\|_{2 N-\ell, \ell}^{2} & \lesssim\|u\|_{0, \ell+2 N-\ell}^{2}+\left\|(\operatorname{curl} u)_{h}\right\|_{2 N-1-\ell, \ell}^{2}+\|\operatorname{div} u\|_{2 N-1-\ell, \ell}^{2} \\
& \leq\|u\|_{0,2 N}^{2}+\left\|(\operatorname{curl} u)_{h}\right\|_{2 N-1-\ell, \ell}^{2}+\left\|\partial_{t} \varrho\right\|_{2 N-1-\ell, \ell}^{2}+\left\|G^{1}\right\|_{2 N-1}^{2} \\
& \lesssim\|u\|_{0,2 N}^{2}+\left\|(\operatorname{curl} u)_{h}\right\|_{2 N-1-\ell, \ell}^{2}+\left\|\partial_{t} \varrho\right\|_{2 N-1}^{2}+\mathfrak{E}_{N+4} \mathcal{E}_{2 N} .
\end{aligned}
$$

On the other hand, consider the following elliptic problem:

$$
\begin{cases}-\kappa \Delta b=\bar{B} \cdot \nabla u-\bar{B} \operatorname{div} u-\partial_{t} b+G^{4} & \text { in } \Omega, \\ b_{3}=0, \kappa \partial_{3} b_{h}=-\bar{B}_{3} u_{h} & \text { on } \partial \Omega .\end{cases}
$$

By the standard $H^{r}$ elliptic estimates with $r=2 N-\ell+1 \geq 2$, the trace theory (as $2 N-\ell \geq 1)$ and (2.19), one has

$$
\begin{aligned}
\|b\|_{2 N-\ell+1, \ell}^{2} & \lesssim\left\|b_{h}\right\|_{0}^{2}+\|u\|_{2 N-\ell, \ell}^{2}+\left\|\partial_{t} b\right\|_{2 N-\ell-1, \ell}^{2}+\left\|G^{4}\right\|_{2 N-\ell-1, \ell}^{2}+\left|u_{h}\right|_{2 N-\frac{1}{2}}^{2} \\
& \lesssim\left\|b_{h}\right\|_{0}^{2}+\|u\|_{2 N-\ell, \ell}^{2}+\left\|\partial_{t} b\right\|_{2 N-1}^{2}+\mathfrak{E}_{N+4} \mathcal{E}_{2 N} .
\end{aligned}
$$

Then it follows from (3.14), (3.15) and (3.17) that

$$
\begin{aligned}
& \frac{d}{d t}\left\|(\operatorname{curl} u)_{h}\right\|_{2 N-1-\ell, \ell}^{2}+\|u\|_{2 N-\ell, \ell}^{2}+\|b\|_{2 N-\ell+1, \ell}^{2} \\
\lesssim & \|u\|_{2 N-1-\ell, \ell+1}^{2}+\|b\|_{2 N-\ell, \ell+1}^{2}+\left\|\partial_{t}(b, \varrho)\right\|_{2 N-1}^{2}+\mathfrak{E}_{N+4} \mathcal{E}_{2 N} .
\end{aligned}
$$


A suitable linear combination of (3.18) for $\ell=0, \ldots, 2 N-1$ yields that

$$
\begin{aligned}
& \frac{d}{d t}\left\|(\operatorname{curl} u)_{h}\right\|_{2 N-1}^{2}+\|u\|_{2 N}^{2}+\|b\|_{2 N+1}^{2} \\
\lesssim & \|u\|_{0,2 N}^{2}+\|b\|_{1,2 N}^{2}+\left\|\partial_{t}(b, \varrho)\right\|_{2 N-1}^{2}+\mathfrak{E}_{N+4} \mathcal{E}_{2 N} .
\end{aligned}
$$

Next, applying curl to the second equation in (2.17) yields

$$
\partial_{t} \operatorname{curl} u=\bar{B} \cdot \nabla \operatorname{curl} b+\operatorname{curl} G^{2} .
$$

For $j=1, \ldots, 2 N-1$, employing the Hodge-type estimates (A.2) of Lemma A.1 with $r=2 N-j \geq 1$, by (3.20), the first equation in (2.17) and (2.19), one obtains

$$
\begin{aligned}
\left\|\partial_{t}^{j} u\right\|_{2 N-j}^{2} \lesssim & \left\|\partial_{t}^{j} u_{h}\right\|_{0}^{2}+\left\|\partial_{t}^{j} \operatorname{curl} u\right\|_{2 N-j-1}^{2}+\left\|\partial_{t}^{j} \operatorname{div} u\right\|_{2 N-j-1}^{2} \\
\lesssim & \left\|\partial_{t}^{j} u_{h}\right\|_{0}^{2}+\left\|\partial_{t}^{j-1} b\right\|_{2 N-j+1}^{2}+\left\|\partial_{t}^{j-1} G^{2}\right\|_{2 N-j}^{2} \\
& +\left\|\partial_{t}^{j+1} \varrho\right\|_{2 N-j-1}^{2}+\left\|\partial_{t}^{j} G^{1}\right\|_{2 N-j-1}^{2} \\
\lesssim & \left\|\partial_{t}^{j} u_{h}\right\|_{0}^{2}+\left\|\partial_{t}^{j-1} b\right\|_{2 N-(j-1)}^{2}+\left\|\partial_{t}^{j+1} \varrho\right\|_{2 N-(j+1)}^{2}+\mathfrak{E}_{N+4} \mathcal{E}_{2 N} .
\end{aligned}
$$

On the other hand, for $j=0, \ldots, 2 N-1$, by the Poincaré inequality and (1.9) and using the second equation in (2.17) and (2.19), one has

$$
\begin{aligned}
\left\|\partial_{t}^{j} \varrho\right\|_{2 N-j}^{2} & \lesssim\left\|\partial_{t}^{j} \nabla \varrho\right\|_{2 N-j-1}^{2} \\
& \lesssim\left\|\partial_{t}^{j+1} u\right\|_{2 N-j-1}^{2}+\left\|\partial_{t}^{j}(\vartheta, b)\right\|_{2 N-j}^{2}+\left\|\partial_{t}^{j} G^{2}\right\|_{2 N-j-1}^{2} \\
& \lesssim\left\|\partial_{t}^{j+1} u\right\|_{2 N-(j+1)}^{2}+\left\|\partial_{t}^{j}(\vartheta, b)\right\|_{2 N-j}^{2}+\mathfrak{E}_{N+4} \mathcal{E}_{2 N} .
\end{aligned}
$$

We shall use the estimates (3.21) and (3.22) repeatedly. First, letting $j=2 N-1$ in (3.21) yields

$$
\left\|\partial_{t}^{2 N-1} u\right\|_{1}^{2} \lesssim\left\|\partial_{t}^{2 N-1} u\right\|_{0}^{2}+\left\|\partial_{t}^{2 N-2} b\right\|_{2}^{2}+\left\|\partial_{t}^{2 N} \varrho\right\|_{0}^{2}+\mathfrak{E}_{N+4} \mathcal{E}_{2 N}
$$

Next, for $j=1, \ldots, 2 N-2,(3.21)$ together with (3.22) (with $j$ replaced by $j+1$ ) implies

$$
\begin{aligned}
\left\|\partial_{t}^{j} u\right\|_{2 N-j}^{2} \lesssim & \left\|\partial_{t}^{j} u\right\|_{0}^{2}+\left\|\partial_{t}^{j-1} b\right\|_{2 N-(j-1)}^{2}+\left\|\partial_{t}^{j+2} u\right\|_{2 N-(j+2)}^{2} \\
& +\left\|\partial_{t}^{j+1}(\vartheta, b)\right\|_{2 N-(j+1)}^{2}+\mathfrak{E}_{N+4} \mathcal{E}_{2 N} .
\end{aligned}
$$


Using a simple induction based on the estimate (3.24), one obtains

$$
\begin{aligned}
\sum_{j=1}^{2 N-2}\left\|\partial_{t}^{j} u\right\|_{2 N-j}^{2} \lesssim & \sum_{j=1}^{2 N-2}\left\|\partial_{t}^{j} u\right\|_{0}^{2}+\sum_{j=0}^{2 N-3}\left\|\partial_{t}^{j} b\right\|_{2 N-j}^{2}+\left\|\partial_{t}^{2 N-1} u\right\|_{1}^{2}+\left\|\partial_{t}^{2 N} u\right\|_{0}^{2} \\
& +\sum_{j=2}^{2 N-1}\left\|\partial_{t}^{j}(\vartheta, b)\right\|_{2 N-j}^{2}+\mathfrak{E}_{N+4} \mathcal{E}_{2 N} .
\end{aligned}
$$

This together with the estimates (3.23) and (3.22) for $j=0, \ldots, 2 N-1$ implies

$$
\begin{aligned}
& \sum_{j=0}^{2 N}\left\|\partial_{t}^{j} \varrho\right\|_{2 N-j}^{2}+\sum_{j=1}^{2 N}\left\|\partial_{t}^{j} u\right\|_{2 N-j}^{2} \\
\lesssim & \sum_{j=1}^{2 N}\left\|\partial_{t}^{j} u\right\|_{0}^{2}+\left\|\partial_{t}^{2 N} \varrho\right\|_{0}^{2}+\sum_{j=0}^{2 N-1}\left\|\partial_{t}^{j}(\vartheta, b)\right\|_{2 N-j}^{2}+\mathfrak{E}_{N+4} \mathcal{E}_{2 N} .
\end{aligned}
$$

Now, applying $\partial_{t}^{j}, j=1, \ldots, 2 N-1$, to the problem (3.16) and the standard $H^{r}$ elliptic estimates with $r=2 N-j+1 \geq 2$, the trace theory (as $2 N-j \geq 1$ ) and (2.19) show that

$$
\begin{aligned}
\left\|\partial_{t}^{j} b\right\|_{2 N-j+1}^{2} \lesssim & \left\|\partial_{t}^{j} b_{h}\right\|_{0}^{2}+\left\|\partial_{t}^{j} u\right\|_{2 N-j}^{2}+\left\|\partial_{t}^{j+1} b\right\|_{2 N-j-1}^{2} \\
& +\left\|\partial_{t}^{j} G^{4}\right\|_{2 N-j-1}^{2}+\left|\partial_{t}^{j} u\right|_{2 N-j-\frac{1}{2}}^{2} \\
\lesssim & \left\|\partial_{t}^{j} b_{h}\right\|_{0}^{2}+\left\|\partial_{t}^{j} u\right\|_{2 N-j}^{2}+\left\|\partial_{t}^{j+1} b\right\|_{2 N-(j+1)}^{2}+\mathfrak{E}_{N+4} \mathcal{E}_{2 N} .
\end{aligned}
$$

On the other hand, consider the following elliptic problem:

$$
\begin{cases}-\mu \Delta \vartheta=-\bar{p} \operatorname{div} u-c_{v} \bar{\rho} \partial_{t} \theta+G^{3} & \text { in } \Omega, \\ \partial_{3} \vartheta=0 & \text { on } \partial \Omega .\end{cases}
$$

Applying $\partial_{t}^{j}, j=0, \ldots, 2 N-1$, to the problem (3.28) and the standard $H^{r}$ elliptic estimates with $r=2 N-j+1 \geq 2$ and (2.19) show that

$$
\begin{aligned}
\left\|\partial_{t}^{j} \vartheta\right\|_{2 N-j+1}^{2} & \lesssim\left\|\partial_{t}^{j} \vartheta\right\|_{0}^{2}+\left\|\partial_{t}^{j} u\right\|_{2 N-j}^{2}+\left\|\partial_{t}^{j+1} \vartheta\right\|_{2 N-j-1}^{2}+\left\|\partial_{t}^{j} G^{3}\right\|_{2 N-j-1}^{2} \\
& \lesssim\left\|\partial_{t}^{j} \vartheta\right\|_{0}^{2}+\left\|\partial_{t}^{j} u\right\|_{2 N-j}^{2}+\left\|\partial_{t}^{j+1} \vartheta\right\|_{2 N-(j+1)}^{2}+\mathfrak{E}_{N+4} \mathcal{E}_{2 N} .
\end{aligned}
$$

Collecting (3.26), (3.27) with summing over $j=1, \ldots, 2 N-1$ and (3.29) with summing over $j=0, \ldots, 2 N-1$ yields that

$$
\sum_{j=0}^{2 N}\left\|\partial_{t}^{j} \varrho\right\|_{2 N-j}^{2}+\sum_{j=1}^{2 N}\left\|\partial_{t}^{j} u\right\|_{2 N-j}^{2}+\sum_{j=0}^{2 N-1}\left\|\partial_{t}^{j} \vartheta\right\|_{2 N-j+1}^{2}+\sum_{j=1}^{2 N-1}\left\|\partial_{t}^{j} b\right\|_{2 N-j+1}^{2}
$$




$$
\lesssim\|u\|_{2 N}^{2}+\sum_{j=1}^{2 N}\left\|\partial_{t}^{j} u\right\|_{0}^{2}+\left\|\partial_{t}^{2 N} \varrho\right\|_{0}^{2}+\sum_{j=0}^{2 N-1}\left\|\partial_{t}^{j}(\vartheta, b)\right\|_{2 N-j}^{2}+\mathfrak{E}_{N+4} \mathcal{E}_{2 N}
$$

Now combining (3.19) and (3.30) leads to

$$
\begin{aligned}
& \frac{d}{d t}\left\|(\operatorname{curl} u)_{h}\right\|_{2 N-1}^{2}+\sum_{j=0}^{2 N}\left\|\partial_{t}^{j}(\varrho, u)\right\|_{2 N-j}^{2}+\sum_{j=0}^{2 N-1}\left\|\partial_{t}^{j}(\vartheta, b)\right\|_{2 N-j+1}^{2} \\
\lesssim & \|u\|_{0,2 N}^{2}+\sum_{j=1}^{2 N}\left\|\partial_{t}^{j} u\right\|_{0}^{2}+\left\|\partial_{t}^{2 N} \varrho\right\|_{0}^{2}+\sum_{j=0}^{2 N-1}\left\|\partial_{t}^{j}(\vartheta, b)\right\|_{2 N-j}^{2}+\|b\|_{1,2 N}^{2}+\mathfrak{E}_{N+4} \mathcal{E}_{2 N} .
\end{aligned}
$$

This together with the Sobolev interpolation implies that

$$
\begin{aligned}
& \frac{d}{d t}\left\|(\operatorname{curl} u)_{h}\right\|_{2 N-1}^{2}+\sum_{j=0}^{2 N}\left\|\partial_{t}^{j}(\varrho, u)\right\|_{2 N-j}^{2}+\sum_{j=0}^{2 N-1}\left\|\partial_{t}^{j}(\vartheta, b)\right\|_{2 N-j+1}^{2} \\
\lesssim & \|u\|_{0,2 N}^{2}+\sum_{j=1}^{2 N}\left\|\partial_{t}^{j} u\right\|_{0}^{2}+\left\|\partial_{t}^{2 N} \varrho\right\|_{0}^{2}+\sum_{j=0}^{2 N-1}\left\|\partial_{t}^{j}(\vartheta, b)\right\|_{0}^{2}+\|b\|_{1,2 N}^{2}+\mathfrak{E}_{N+4} \mathcal{E}_{2 N},
\end{aligned}
$$

which yields (3.11) by controlling the first fourth terms in the right hand side by $\overline{\mathcal{E}}_{2 N}$ and the fifth term by $\overline{\mathcal{D}}_{2 N}$.

We now prove (3.12). Recalling (3.15) with $\ell=0,(3.26)$, (3.27) and (3.29) for $j=0, \ldots, 2 N-1$, and then summing them up, similarly as the derivation of (3.32), one deduces

$$
\begin{aligned}
\mathcal{E}_{2 N} \lesssim & \|u\|_{0,2 N}^{2}+\sum_{j=1}^{2 N}\left\|\partial_{t}^{j} u\right\|_{0}^{2}+\left\|\partial_{t}^{2 N} \varrho\right\|_{0}^{2}+\sum_{j=0}^{2 N-1}\left\|\partial_{t}^{j}(\vartheta, b)\right\|_{0}^{2} \\
& +\left\|(\operatorname{curl} u)_{h}\right\|_{2 N-1}^{2}+\mathfrak{E}_{N+4} \mathcal{E}_{2 N},
\end{aligned}
$$

which yields (3.12) since the first four terms in the right hand side can be controlled by $\overline{\mathcal{E}}_{2 N}$.

We now deduce the boundedness estimates of $\mathcal{E}_{2 N}$. Set

$$
\mathfrak{E}_{N+4}^{w}(t):=(1+t)^{N-5} \mathfrak{E}_{N+4}(t) .
$$

Theorem 3.1. Let $N \geq 8$. It holds that

$$
\mathcal{E}_{2 N}(t)+\int_{0}^{t} \overline{\mathcal{D}}_{2 N}(s) d s \lesssim \mathcal{E}_{2 N}(0)+\sup _{0 \leq s \leq t} \mathcal{E}_{2 N}(s) \sqrt{\mathfrak{E}_{N+4}^{w}(s)}, \quad \forall t \in[0, T] .
$$


Proof. First, integrating (2.8) in time implies that for $N \geq 8$,

$$
\begin{aligned}
& \overline{\mathcal{E}}_{2 N}(t)+\int_{0}^{t} \overline{\mathcal{D}}_{2 N}(s) d s \\
\lesssim & \overline{\mathcal{E}}_{2 N}(0)+\sup _{0 \leq s \leq t} \mathcal{E}_{2 N}(s) \sqrt{\mathfrak{E}_{N+4}^{w}(s)} \int_{0}^{t}(1+s)^{-(N-5) / 2} d s \\
\lesssim & \mathcal{E}_{2 N}(0)+\sup _{0 \leq s \leq t} \mathcal{E}_{2 N}(s) \sqrt{\mathfrak{E}_{N+4}^{w}(s)} .
\end{aligned}
$$

Next, a Gronwall type argument for (3.11) yields

$$
\begin{aligned}
\left\|(\operatorname{curl} u)_{h}(t)\right\|_{2 N-1}^{2} \lesssim & \mathcal{E}_{2 N}(0)+\sup _{0 \leq s \leq t} \overline{\mathcal{E}}_{2 N}(s)+\int_{0}^{t} \overline{\mathcal{D}}_{2 N}(s) d s \\
& +\sup _{0 \leq s \leq t}\left(\mathcal{E}_{2 N}(s) \mathcal{E}_{N+4}(s)\right)^{2} .
\end{aligned}
$$

Hence, one concludes (3.35) from (3.36), (3.37) and (3.12).

\section{Decay estimates of $\mathfrak{E}_{N+4}$}

In this section, to close the full energy estimates (3.35), we will derive the decay estimates of $\mathfrak{E}_{N+4}$. This will follow from a set of energy-dissipation estimates related to $\mathfrak{E}_{n}$ and $\mathfrak{D}_{n}$.

Note that the tangential dissipation estimates of $\overline{\mathcal{D}}_{n}$ control only the temperature $\vartheta$ and magnetic field $b$. The tangential dissipation estimates for the velocity $u$ rely on the coupling between the fluid and the magnetic field and the presence of the heat conductivity, and one has the following.

Proposition 4.1. For $n=N+4, \ldots, 2 N$, it holds that

$$
\sum_{j=0}^{n-1}\left\|\partial_{t}^{j} u\right\|_{0, n-j-1}^{2} \lesssim \overline{\mathcal{D}}_{n}+\mathfrak{D}_{N+4} \mathcal{E}_{2 N}
$$

Proof. Let $n=N+4, \ldots, 2 N$. It follows from the fourth and third equations in (2.17) that

$$
\partial_{t} b-\kappa \Delta b=\bar{B} \cdot \nabla u+\frac{\bar{B}}{\bar{p}}\left(c_{v} \bar{\rho} \partial_{t} \vartheta-\mu \Delta \vartheta-G^{3}\right)+G^{4} .
$$

By the vertical component of (4.2) and using the fifth equation in (2.17), one obtains

$$
\bar{B} \cdot \nabla\left(u_{3}-\frac{\mu}{\bar{p}} \partial_{3} \vartheta\right) \equiv \bar{B}_{h} \cdot \nabla_{h}\left(-\frac{\mu}{\bar{p}} \partial_{3} \vartheta\right)+\bar{B} \cdot \nabla u_{3}-\frac{\mu \bar{B}_{3}}{\bar{p}} \partial_{3}^{2} \vartheta
$$




$$
=\bar{B}_{h} \cdot \nabla_{h}\left(-\frac{\mu}{\bar{p}} \partial_{3} \vartheta\right)+\partial_{t} b_{3}-\kappa \Delta_{h} b_{3}+\kappa \partial_{3} \operatorname{div}_{h} b_{h}-\frac{\bar{B}_{3}}{\bar{p}}\left(c_{v} \bar{\rho} \partial_{t} \vartheta-\mu \Delta_{h} \vartheta-G^{3}\right)-G_{3}^{4} .
$$

As $\bar{B}_{3} \neq 0$ and since $u_{3}=0$ and $\partial_{3} \vartheta=0$ on $\partial \Omega$, by the Poincaré-type inequality, (4.3) and (2.19), one deduces that for $j=0, \ldots, n-1$,

$$
\begin{aligned}
& \left\|\partial_{t}^{j}\left(u_{3}-\frac{\mu}{\bar{p}} \partial_{3} \vartheta\right)\right\|_{0, n-j-1}^{2} \lesssim\left\|\bar{B} \cdot \nabla \partial_{t}^{j}\left(u_{3}-\frac{\mu}{\bar{p}} \partial_{3} \vartheta\right)\right\|_{0, n-j-1}^{2} \\
\lesssim & \left\|\partial_{t}^{j}(\vartheta, b)\right\|_{1, n-j}^{2}+\left\|\partial_{t}^{j+1}\left(\vartheta, b_{3}\right)\right\|_{0, n-j-1}^{2}+\left\|\partial_{t}^{j} G^{3}\right\|_{n-j-1}^{2}+\left\|\partial_{t}^{j} G_{3}^{4}\right\|_{n-j-1}^{2} \\
\lesssim & \overline{\mathcal{D}}_{n}+\mathfrak{D}_{N+4} \mathcal{E}_{2 N} .
\end{aligned}
$$

This implies

$$
\begin{aligned}
\left\|\partial_{t}^{j} u_{3}\right\|_{0, n-j-1}^{2} & \lesssim\left\|\partial_{t}^{j}\left(u_{3}-\frac{\mu}{\bar{p}} \partial_{3} \vartheta\right)\right\|_{0, n-j-1}^{2}+\left\|\partial_{t}^{j} \vartheta\right\|_{1, n-j-1}^{2} \\
& \lesssim \overline{\mathcal{D}}_{n}+\mathfrak{D}_{N+4} \mathcal{E}_{2 N} .
\end{aligned}
$$

Now by the horizontal components of (4.2), one finds

$$
\begin{aligned}
& \bar{B} \cdot \nabla\left(\kappa \partial_{3} b_{h}+\bar{B}_{3} u_{h}-\frac{\mu \bar{B}_{h}}{\bar{p}} \partial_{3} \vartheta\right) \\
\equiv & \bar{B}_{h} \cdot \nabla_{h}\left(\kappa \partial_{3} b_{h}-\frac{\mu \bar{B}_{h}}{\bar{p}} \partial_{3} \vartheta\right)+\bar{B}_{3}\left(\kappa \partial_{3}^{2} b_{h}+\bar{B} \cdot \nabla u_{h}-\frac{\mu \bar{B}_{h}}{\bar{p}} \partial_{3}^{2} \vartheta\right) \\
= & \bar{B}_{h} \cdot \nabla_{h}\left(\kappa \partial_{3} b_{h}-\frac{\mu \bar{B}_{h}}{\bar{p}} \partial_{3} \vartheta\right)+\bar{B}_{3}\left(\partial_{t} b_{h}-\kappa \Delta_{h} b_{h}-\frac{\bar{B}_{h}}{\bar{p}}\left(c_{v} \bar{\rho} \partial_{t} \vartheta-\mu \Delta_{h} \vartheta-G^{3}\right)-G_{h}^{4}\right) .
\end{aligned}
$$

Since $\partial_{3} \vartheta=0$ and $\kappa \partial_{3} b_{h}+\bar{B}_{3} u_{h}=0$ on $\partial \Omega$, by the Poincaré-type inequality again, (4.6) and (2.19), one deduces that for $j=0, \ldots, n-1$,

$$
\begin{aligned}
& \left\|\partial_{t}^{j}\left(\kappa \partial_{3} b_{h}+\bar{B}_{3} u_{h}-\frac{\mu \bar{B}_{h}}{\bar{p}} \partial_{3} \vartheta\right)\right\|_{0, n-j-1}^{2} \\
\lesssim & \left\|\bar{B} \cdot \nabla \partial_{t}^{j}\left(\kappa \partial_{3} b_{h}+\bar{B}_{3} u_{h}-\frac{\mu \bar{B}_{h}}{\bar{p}} \partial_{3} \vartheta\right)\right\|_{0, n-j-1}^{2} \\
\lesssim & \left\|\partial_{t}^{j}\left(\vartheta, b_{h}\right)\right\|_{1, n-j}^{2}+\left\|\partial_{t}^{j+1}\left(\vartheta, b_{h}\right)\right\|_{0, n-j-1}^{2}+\left\|\partial_{t}^{j} G^{3}\right\|_{n-j-1}^{2}+\left\|\partial_{t}^{j} G_{h}^{4}\right\|_{n-j-1}^{2} \\
\lesssim & \overline{\mathcal{D}}_{n}+\mathfrak{D}_{N+4} \mathcal{E}_{2 N} .
\end{aligned}
$$


This implies, using $\bar{B}_{3} \neq 0$ again, that

$$
\begin{aligned}
\left\|\partial_{t}^{j} u_{h}\right\|_{0, n-j-1}^{2} & \lesssim\left\|\partial_{t}^{j}\left(\kappa \partial_{3} b_{h}+\bar{B}_{3} u_{h}-\frac{\mu \bar{B}_{h}}{\bar{p}} \partial_{3} \vartheta\right)\right\|_{0, n-j-1}^{2}+\left\|\partial_{t}^{j}\left(\vartheta, b_{h}\right)\right\|_{1, n-j-1}^{2} \\
& \lesssim \overline{\mathcal{D}}_{n}+\mathfrak{D}_{N+4} \mathcal{E}_{2 N} .
\end{aligned}
$$

This together with (4.5) gives (4.1).

Next, we derive the dissipation estimates of $\partial_{t}^{n-1} \varrho$.

Proposition 4.2. For $n=N+4, \ldots, 2 N$, it holds that

$$
\frac{d}{d t} \int_{\Omega} \bar{\rho} \partial_{t}^{n-1} u \cdot \partial_{t}^{n-2} u+\left\|\partial_{t}^{n-1} \varrho\right\|_{0}^{2} \lesssim \overline{\mathcal{D}}_{n}+\sqrt{\mathcal{E}_{2 N}} \mathfrak{D}_{n}
$$

Proof. Let $n=N+4, \ldots, 2 N$. One applies $\partial_{t}^{n-2}$ to the first equation in (2.17) and then takes the inner product with $\frac{R \bar{\theta}}{\bar{\rho}} \partial_{t}^{n-1} \varrho$ to have

$$
\int_{\Omega} \frac{R \bar{\theta}}{\bar{\rho}}\left|\partial_{t}^{n-1} \varrho\right|^{2}+\int_{\Omega} R \bar{\theta} \partial_{t}^{n-1} \varrho \operatorname{div} \partial_{t}^{n-2} u=\int_{\Omega} \frac{R \bar{\theta}}{\bar{\rho}} \partial_{t}^{n-1} \varrho \partial_{t}^{n-2} G^{1} .
$$

By (2.19), one obtains

$$
\int_{\Omega} \frac{R \bar{\theta}}{\bar{\rho}} \partial_{t}^{n-1} \varrho \partial_{t}^{n-2} G^{1} \lesssim\left\|\partial_{t}^{n-1} \varrho\right\|_{0}\left\|\partial_{t}^{n-2} G^{1}\right\|_{0} \lesssim \sqrt{\mathfrak{D}_{n}} \sqrt{\mathfrak{D}_{N+4} \mathcal{E}_{2 N}}
$$

Integrating by parts over $\Omega$ and using the second equation in (2.17), one has

$$
\begin{aligned}
& \int_{\Omega} R \bar{\theta} \partial_{t}^{n-1} \varrho \operatorname{div} \partial_{t}^{n-2} u=-\int_{\Omega} \nabla\left(R \bar{\theta} \partial_{t}^{n-1} \varrho\right) \cdot \partial_{t}^{n-2} u \\
= & \int_{\Omega}\left(\bar{\rho} \partial_{t}^{n} u+\nabla\left(R \bar{\rho} \partial_{t}^{n-1} \vartheta\right)-\operatorname{curl} \partial_{t}^{n-1} b \times \bar{B}-\partial_{t}^{n-1} G^{2}\right) \cdot \partial_{t}^{n-2} u .
\end{aligned}
$$

Integrating by parts in time yields

$$
\int_{\Omega} \bar{\rho} \partial_{t}^{n} u \cdot \partial_{t}^{n-2} u=\frac{d}{d t} \int_{\Omega} \bar{\rho} \partial_{t}^{n-1} u \cdot \partial_{t}^{n-2} u-\int_{\Omega} \bar{\rho}\left|\partial_{t}^{n-1} u\right|^{2} .
$$

One obtains directly

$$
-\int_{\Omega}\left(\nabla\left(R \bar{\rho} \partial_{t}^{n-1} \vartheta\right)-\operatorname{curl} \partial_{t}^{n-1} b \times \bar{B}\right) \cdot \partial_{t}^{n-2} u \lesssim\left\|\partial_{t}^{n-1}(\vartheta, b)\right\|_{1}\left\|\partial_{t}^{n-2} u\right\|_{0}
$$


and by (2.19),

$$
\int_{\Omega} \partial_{t}^{n-1} G^{2} \cdot \bar{\rho} \partial_{t}^{n-2} u \lesssim\left\|\partial_{t}^{n-1} G^{2}\right\|_{0}\left\|\partial_{t}^{n-2} u\right\|_{0} \lesssim \sqrt{\mathfrak{D}_{N+4} \mathcal{E}_{2 N}} \sqrt{\mathfrak{D}_{n}}
$$

Consequently, combining (4.10)-(4.15) yields, by Cauchy's inequality,

$$
\begin{aligned}
& \frac{d}{d t} \int_{\Omega} \bar{\rho} \partial_{t}^{n-1} u \cdot \partial_{t}^{n-2} u+\left\|\partial_{t}^{n-1} \varrho\right\|_{0}^{2} \\
\lesssim & \left\|\left(\partial_{t}^{n-2} u, \partial_{t}^{n-3} u\right)\right\|_{0}^{2}+\left\|\partial_{t}^{n-1}(\vartheta, b)\right\|_{1}^{2}+\sqrt{\mathcal{E}_{2 N}} \mathfrak{D}_{n} .
\end{aligned}
$$

One thus concludes (4.9) by controlling the first term in the right hand side by using (4.1) and the second term by $\overline{\mathcal{D}}_{n-1}$, since $n \geq N+4$.

Now we derive the full energy-dissipation estimates.

Proposition 4.3. For $n=N+4, \ldots, 2 N$, it holds that

$$
\frac{d}{d t}\left(\left\|(\operatorname{curl} u)_{h}\right\|_{n-2}^{2}+\int_{\Omega} \bar{\rho} \partial_{t}^{n-1} u \cdot \partial_{t}^{n-2} u\right)+\mathfrak{D}_{n} \lesssim \overline{\mathcal{D}}_{n}+\sqrt{\mathcal{E}_{2 N}} \mathfrak{D}_{n}
$$

and that

$$
\mathfrak{E}_{n} \lesssim \overline{\mathcal{E}}_{n}+\left\|(\operatorname{curl} u)_{h}\right\|_{n-2}^{2}+\mathfrak{E}_{N+4} \mathcal{E}_{2 N}
$$

Proof. Let $n=N+4, \ldots, 2 N$. It follows similarly as the derivation of (3.32), with $2 N$ replaced by $n-1$, that

$$
\begin{aligned}
& \frac{d}{d t}\left\|(\operatorname{curl} u)_{h}\right\|_{n-2}^{2}+\sum_{j=0}^{n-1}\left\|\partial_{t}^{j}(\varrho, u)\right\|_{n-j-1}^{2}+\sum_{j=0}^{n-2}\left\|\partial_{t}^{j}(\vartheta, b)\right\|_{n-j}^{2} \\
\lesssim & \|u\|_{0, n-1}^{2}+\sum_{j=1}^{n-1}\left\|\partial_{t}^{j} u\right\|_{0}^{2}+\left\|\partial_{t}^{n-1} \varrho\right\|_{0}^{2}+\sum_{j=0}^{n-2}\left\|\partial_{t}^{j}(\vartheta, b)\right\|_{0}^{2}+\|b\|_{1, n-1}^{2}+\mathfrak{D}_{N+4} \mathcal{E}_{2 N} .
\end{aligned}
$$

One may appeal to (4.1) to control the first two terms in the right hand side, and the fourth and fifth terms are bounded by $\overline{\mathcal{D}}_{n-2}$. One then combines the resulting inequality and (4.9) to conclude (4.17).

We now prove (4.18). Taking $\ell=0$ and replacing $2 N$ by $n-1$ in (3.15), taking $j=0$ and replacing $2 N$ by $n-1$ in (3.27) and (3.29), and then combining them yield

$$
\begin{gathered}
\|u\|_{n-1}^{2}+\|(\vartheta, b)\|_{n}^{2} \lesssim\|u\|_{0, n-1}^{2}+\left\|(\operatorname{curl} u)_{h}\right\|_{n-2}^{2}+\|(\vartheta, b)\|_{0}^{2} \\
+\left\|\partial_{t}(\varrho, \vartheta, b)\right\|_{n-2}^{2}+\mathfrak{E}_{N+4} \mathcal{E}_{2 N} .
\end{gathered}
$$


Replacing $2 N$ by $n-1$ in (3.30) (but without estimating $\|\vartheta\|_{n}^{2}$ ) leads to

$$
\begin{aligned}
& \sum_{j=0}^{n}\left\|\partial_{t}^{j} \varrho\right\|_{n-j}^{2}+\sum_{j=1}^{n}\left\|\partial_{t}^{j} u\right\|_{n-j}^{2}+\sum_{j=1}^{n-1}\left\|\partial_{t}^{j}(\vartheta, b)\right\|_{n-j+1}^{2} \\
\lesssim & \sum_{j=1}^{n}\left\|\partial_{t}^{j} u\right\|_{0}^{2}+\left\|\partial_{t}^{n} \varrho\right\|_{0}^{2}+\sum_{j=0}^{n-1}\left\|\partial_{t}^{j}(\vartheta, b)\right\|_{2 N-j}^{2}+\mathfrak{E}_{N+4} \mathcal{E}_{2 N} .
\end{aligned}
$$

Hence, combining (4.20)-(4.21) gives

$$
\mathfrak{E}_{n} \lesssim \overline{\mathcal{E}}_{n}+\left\|(\operatorname{curl} u)_{h}\right\|_{n-2}^{2}+\left\|\partial_{t} \varrho\right\|_{n-2}^{2}+\sum_{j=0}^{n-1}\left\|\partial_{t}^{j}(\vartheta, b)\right\|_{n-j}^{2}+\mathfrak{E}_{N+4} \mathcal{E}_{2 N}
$$

This together with the Sobolev interpolation implies that

$$
\begin{aligned}
\mathfrak{E}_{n} & \lesssim \overline{\mathcal{E}}_{n}+\left\|(\operatorname{curl} u)_{h}\right\|_{n-2}^{2}+\left\|\partial_{t} \varrho\right\|_{0}^{2}+\sum_{j=0}^{n-1}\left\|\partial_{t}^{j}(\vartheta, b)\right\|_{0}^{2}+\mathfrak{E}_{N+4} \mathcal{E}_{2 N} \\
& \lesssim \overline{\mathcal{E}}_{n}+\left\|(\operatorname{curl} u)_{h}\right\|_{n-2}^{2}+\mathfrak{E}_{N+4} \mathcal{E}_{2 N}
\end{aligned}
$$

This is (4.18).

With the previous estimates in hand, we now derive the decay estimates.

Theorem 4.1. It holds that

$$
\begin{aligned}
& \int_{0}^{t} \mathfrak{D}_{2 N}(s) d s+\sum_{j=0}^{N-6}(1+t)^{N-5-j} \mathfrak{E}_{N+4+j}(t)+\sum_{j=0}^{N-6} \int_{0}^{t}(1+s)^{N-5-j} \mathfrak{D}_{N+4+j}(s) d s \\
\lesssim & \mathcal{E}_{2 N}(0)+\sup _{0 \leq s \leq t} \mathcal{E}_{2 N}(s) \sqrt{\mathfrak{E}_{N+4}^{w}(s)} .
\end{aligned}
$$

Proof. First, taking $n=2 N$ in (4.17), one deduces that for $\mathcal{E}_{2 N} \leq \delta$ small,

$$
\frac{d}{d t}\left(\left\|(\operatorname{curl} u)_{h}\right\|_{2 N-2}^{2}+\int_{\Omega} \bar{\rho} \partial_{t}^{2 N-1} u \cdot \partial_{t}^{2 N-2} u\right)+\mathfrak{D}_{2 N} \lesssim \overline{\mathcal{D}}_{2 N}
$$

Integrating (4.25) in time and by (3.35) gives in particular that

$$
\begin{aligned}
\int_{0}^{t} \mathfrak{D}_{2 N}(s) d s & \lesssim \mathcal{E}_{2 N}(0)+\overline{\mathcal{E}}_{2 N}(t)+\int_{0}^{t} \overline{\mathcal{D}}_{2 N}(s) d s \\
& \lesssim \mathcal{E}_{2 N}(0)+\sup _{0 \leq s \leq t} \mathcal{E}_{2 N}(s) \sqrt{\mathfrak{E}_{N+4}^{w}(s)} .
\end{aligned}
$$


Next, it follows from (4.17) that for $n=N+4, \ldots, 2 N-2$,

$$
\frac{d}{d t}\left(\left\|(\operatorname{curl} u)_{h}\right\|_{n-2}^{2}+\int_{\Omega} \bar{\rho} \partial_{t}^{n-1} u \cdot \partial_{t}^{n-2} u\right)+\mathfrak{D}_{n} \lesssim \overline{\mathcal{D}}_{n} .
$$

On the other hand, it follows from (4.18) that

$$
\mathfrak{E}_{n} \lesssim \overline{\mathcal{E}}_{n}+\left\|(\operatorname{curl} u)_{h}\right\|_{n-2}^{2} .
$$

One can deduce from (4.27), (4.28), (2.20) and (2.21) that

$$
\frac{d}{d t} \mathfrak{E}_{n}+\mathfrak{D}_{n} \leq 0
$$

Observe that $\mathfrak{E}_{\ell} \leq \mathfrak{D}_{\ell+1}$. Then we will employ a time weighted inductive argument here. To begin with, it follows from (4.29) that for $j=0, \ldots, N-4$,

$$
\frac{d}{d t} \mathfrak{E}_{N+4+j}+\mathfrak{D}_{N+4+j} \leq 0 .
$$

Multiplying (4.30) by $(1+t)^{N-5-j}$ and using $\mathfrak{E}_{N+4+j} \leq \mathfrak{D}_{N+5+j}$, one gets

$$
\begin{aligned}
& \frac{d}{d t}\left((1+t)^{N-5-j} \mathfrak{E}_{N+4+j}\right)+(1+t)^{N-5-j} \mathfrak{D}_{N+4+j} \\
\leq & (N-5-j)(1+t)^{N-6-j} \mathfrak{E}_{N+4+j} \lesssim(1+t)^{N-5-(j+1)} \mathfrak{D}_{N+4+(j+1)} .
\end{aligned}
$$

Integrating (4.31) in time directly and using a suitable linear combination of the resulting inequalities, one obtains

$$
\begin{aligned}
& \sum_{j=0}^{N-6}(1+t)^{N-5-j} \mathfrak{E}_{N+4+j}(t)+\sum_{j=0}^{N-6} \int_{0}^{t}(1+s)^{N-5-j} \mathfrak{D}_{N+4+j}(s) d s \\
\lesssim & \mathcal{E}_{2 N}(0)+\int_{0}^{t} \mathfrak{D}_{2 N-1}(s) d s .
\end{aligned}
$$

This together with (4.26) implies (4.24).

\section{Global well-posedness}

We are now ready to present the proof of Theorem 1.1.

Proof. The estimate (4.24) implies in particular (1.16), for $\mathcal{E}_{2 N} \leq \delta$ small. Then combining the estimates (3.35) and (1.16) yields (1.15). This closes the a priori assumption (2.1) if $\mathcal{E}_{2 N}(0) \leq \varepsilon_{0}$ for $\varepsilon_{0}>0$ sufficiently small. Therefore, the global well-posedness follows by using a standard continuity argument. 


\section{Appendix A. Hodge-type estimates}

Lemma A.1. Let $r \geq 1$ be an integer. Then it holds that

$$
\|v\|_{r} \lesssim\|v\|_{0, r}+\left\|(\operatorname{curl} v)_{h}\right\|_{r-1}+\|\operatorname{div} v\|_{r-1} .
$$

If $v_{3}=0$ on $\partial \Omega$, then

$$
\|v\|_{r} \lesssim\left\|v_{h}\right\|_{0}+\|\operatorname{curl} v\|_{r-1}+\|\operatorname{div} v\|_{r-1} .
$$

If $v_{3}=0$ on $\partial \Omega$ and $\int_{\Omega} v_{h}=0$, then

$$
\|v\|_{r} \lesssim\|\operatorname{curl} v\|_{r-1}+\|\operatorname{div} v\|_{r-1} .
$$

Proof. One may refer to [21, Lemma A.9] for the proof of (A.1). Now for $v_{3}=0$ on $\partial \Omega$, by the standard elliptic estimates on $-\Delta v_{3}=(\text { curlcurl } v)_{3}-\partial_{3} \operatorname{div} v$, one obtains

$$
\left\|v_{3}\right\|_{r} \lesssim\left\|(\operatorname{curl} v)_{h}\right\|_{r-1}+\|\operatorname{div} v\|_{r-1} .
$$

On the other hand, one has

$$
\left\|\nabla v_{h}\right\|_{r-1} \lesssim\left\|\nabla v_{3}\right\|_{r-1}+\|\operatorname{curl} v\|_{r-1}+\|\operatorname{div} v\|_{r-1} .
$$

Then (A.2) follows from (A.4)-(A.5), and (A.3) follows by using further the Poincaré inequality.

\section{Acknowledgements}

Y. J. Wang was supported by the National Natural Science Foundation of China $(11771360,12171401)$ and the Natural Science Foundation of Fujian Province of China (2019J02003). Z. P. Xin was supported by Zheng Ge Ru Foundation, Hong Kong RGC Earmarked Research Grants CUHK14305315, CUHK14302819, CUHK14300917, CUHK14302917, CUHK14300819, and Basic and Applied Basic Research Foundation of Guangdong Province (2020B1515310002).

\section{References}

[1] H. Abidi and P. Zhang, On the global solution of a 3-D MHD system with initial data near equilibrium, Comm. Pure Appl. Math. 70(8) (2017), 1509-1561. 
[2] C. Bardos, C. Sulem, and P.-L. Sulem, Longtime dynamics of a conductive fluid in the presence of a strong magnetic field, Trans. Amer. Math. Soc. 305(1) (1988), 175-191.

[3] Y. Cai and Z. Lei, Global well-posedness of the incompressible magnetohydrodynamics, Arch. Ration. Mech. Anal. 228(3) (2018), 969-993.

[4] C. Cao and J. Wu, Global regularity for the 2D MHD equations with mixed partial dissipation and magnetic diffusion, Adv. Math. 226(2) (2011), 1803-1822.

[5] G. Duvaut and J. L. Lions, Inéquations en thermoélasticité et magnétohydrodynamique, Arch. Ration. Mech. Anal. 46 (1972), 241-279.

[6] L. C. Evans, Partial Differential Equations. Second Edition, Graduate Studies in Mathematics 19, AMS, 2010.

[7] J. Goedbloed and S. Poedts, Principles of Magnetohydrodynamics with Applications to Laboratory and Astrophysical Plasmas, Cambridge University Press, 2004.

[8] L. He, L. Xu and P. Yu, On global dynamics of three dimensional magnetohydrodynamics: nonlinear stability of Alfvén waves, Ann. PDE 4(1) (2018), Paper No. 5, 105 pp.

[9] S. Kawashima, System of a Hyperbolic-Parabolic Composite Type, with Applications to the Equations of Magnetohydrodynamics, Ph.D. Thesis, Kyoto University, 1984.

[10] H. Kozono, Weak and classical solutions of the two-dimensional magnetohydrodynamic equations, Tohoku Math. J. (2) 41(3) (1989), 471-488.

[11] L. D. Landau and E. M. Lifshitz, Electrodynamics of Continuous Media, Pergamon, 1984.

[12] Z. Lei and Y. Zhou, BKM's criterion and global weak solutions for magnetohydrodynamics with zero viscosity, Discrete Contin. Dyn. Syst. 25(2) (2009), 575-583.

[13] F. Lin, L. Xu and P. Zhang, Global small solutions of 2-D incompressible MHD system, J. Differ. Eq. 259(10) (2015), 5440-5485.

[14] T.-P. Liu and Y. Zeng, Large time behavior of solutions for general quasilinear hyperbolicparabolic systems of conservation laws, Mem. Amer. Math. Soc. 125(599) (1997), viii+ $120 \mathrm{pp}$.

[15] X. Ren, J. Wu, Z. Xiang and Z. Zhang, Global existence and decay of smooth solution for the 2-D MHD equations without magnetic diffusion, J. Funct. Anal. 267(2) (2014), 503-541.

[16] X. Ren, Z. Xiang and Z. Zhang, Global well-posedness of 2-D MHD equations without magnetic diffusion in a strip domain, Nonlinearity 29(4) (2016), 1257-1291.

[17] S. Schochet, The compressible Euler equations in a bounded domain: existence of solutions and the incompressible limit, Comm. Math. Phys. 104(1) (1986), 49-75.

[18] M. Sermange and R. Temam, Some mathematical questions related to the MHD equations, Comm. Pure Appl. Math. 36(5) (1983), 635-664.

[19] M. Slemrod, Global existence, uniqueness, and asymptotic stability of classical smooth solutions in one-dimensional nonlinear thermoelasticity, Arch. Ration. Mech. Anal. 76(2) (1981), 97-133.

[20] Z. Tan and Y. J. Wang, Global well-posedness of an initial-boundary value problem for 
viscous non-resistive MHD systems, SIAM J. Math. Anal. 50(1) (2018), 1432-1470.

[21] Y. J. Wang and Z. Xin, Global well-posedness of free interface problems for the incompressible inviscid resistive $M H D$, arXiv: 2009.11636v1.

[22] D. Wei and Z. Zhang, Global well-posedness of the MHD equations in a homogeneous magnetic field, Anal. PDE 10(6) (2017), 1361-1406.

[23] D. Wei and Z. Zhang, Global well-posedness for the 2-D MHD equations with magnetic diffusion, Commun. Math. Res. 36(4) (2020), 377-389.

[24] J. Wu and Y. Wu, Global small solutions to the compressible 2D magnetohydrodynamic system without magnetic diffusion, Adv. Math. 310 (2017), 759-888.

[25] L. Xu and P. Zhang, Global small solutions to three-dimensional incompressible magnetohydrodynamical system, SIAM J. Math. Anal. 47(1) (2015), 26-65.

[26] Y. Zeng, Global existence theory for general hyperbolic-parabolic balance laws with application, J. Hyperbolic Differ. Equ. 14(2) (2017), 359-391.

[27] T. Zhang, Global solutions to the 2D viscous, non-resistive MHD system with large background magnetic field, J. Differ. Equ. 260(6) (2016), 5450-5480.

[28] Y. Zhou and Y. Zhu, Global classical solutions of 2D MHD system with only magnetic diffusion on periodic domain, J. Math. Phys. 59(8) (2018), 081505, 12 pp. 